\title{
COVID-19, Your Pet and Other Animals: Are You at Risk?
}

\section{María Irian Percedo-Abreu DVM PhD}

Despite fast-tracked research, the precise origin, transmission and evolution of COVID-19 are still unknown. While the bat genus Rhinolophus is likely the primary source of the zoonotic-origin pathogen SARS-CoV-2 that causes COVID-19, its transmission route into the human population is still being studied.[1,2]

Coronaviruses (CoV) affect humans and various animal species. Bats were the original hosts of the CoV that causes Severe Acute Respiratory Syndrome (SARS-CoV) and Middle East Respiratory Syndrome coronavirus (MERS-CoV), for example, with masked palm civet cats and dromedaries, respectively, the intermediate hosts of those two viruses. Research is ongoing regarding intermediate species for SARS-CoV-2, but one possibility is the large stray cat and dog population around the live animal market in Wuhan, China, where the pandemic is thought to have started.

Explaining the epidemiological role of different species in COVID-19 transmission is fundamental for its prevention and control, since identifying possible viral reservoirs can limit future, unexpected outbreaks. For example, MERS control included a proposal for vaccinating dromedaries;[3] surveillance of SARS-CoV-2 in undomesticated cats and dogs can serve a similar purpose.[4,5]

After asymptomatic dogs were infected with SARS-CoV-2 following close contact with their infected owners in Hong Kong in March, 2020, the World Organization for Animal Health (OIE) began requiring notification of domestic animals infected with SARS-CoV-2. An ad hoc group of international experts was convened to analyze reports and undertake research to better understand the epidemiological significance for animal health, biodiversity and public health.[1]

In experimental studies, pigs and poultry did not become infected with SARS-CoV-2, but ferrets and cats did; dogs were less susceptible. In silico models suggest other mammals that might become infected like humans include chimpanzees, monkeys and bovines, given the similarity in angiotensin-converting enzyme 2 (ACE2) receptors, with high affinity for the spike (S) glycoprotein.[4]

Cats, especially, require close surveillance given how efficiently the SARS-CoV-2 virus multiplies in experimental inoculations. Even asymptomatic cats transmitted the virus to other cats via aerosol a few days after being exposed. Outdoor cats from homes where COVID-19 is present may transmit the disease, as well as those having contact with potentially contaminated wastewater from affected communities.

There have been numerous reports of cats naturally infected in several European countries, Hong Kong, Russia and the United States, following close contact with their sick owners. Active surveillance of 102 cats in Wuhan during the epidemic revealed 15 $(14.7 \%)$ were seropositive for SARS-CoV-2. The highest titers were found in 3 cats whose owners were confirmed with COVID-19; of the remaining 12, 6 came from a pet shelter and the other 6 were street cats.[6] Symptomatic lions and tigers that tested positive for COVID-19 at the Bronx Zoo in New York is another indication of feline susceptibility.
A study of 817 pets (540 dogs, 277 cats) in Italy when the country was experiencing high rates of the epidemic, revealed none tested positive for COVID-19 using real-time polymerase chain reaction (RT-PCR). However, 3.4\% dogs and 3.9\% cats showed a prevalence of SARS-CoV-2-neutralizing antibody titers-similar to rates in the human population-with a higher probability for those dogs from homes with owners testing positive for COVID-19.[7]

Meanwhile, dozens of mink farms in the Netherlands, Denmark and Spain and two in the USA have been infected with SARSCoV-2. Clinical signs varied in the Netherlands, where thousands of mink were sacrificed and reverse zoonosis (human to mink) of SARS-CoV-2, with further transmission from farmworkers to people in their homes, was documented. Stray cats and dogs in the vicinity of the infected farms also tested positive. A total mink farm ban goes into effect in that country in early 2021 to avoid potential viral reservoirs.

Farm animals have greater
potential to become viral
reservoirs due to
industrial-scale farming
practices

Susceptibility to SARSCoV-2 in rabbits is another concern. Farm animals have greater potential to become viral reservoirs due to industrial-scale farming practices with large numbers of animals in continual production. Such conditions lend themselves to longer outbreaks with higher viral loads, since newborns are more prone to infection and facilitate transmission. Furthermore, asymptomatic animals contribute to low risk perception. Strict controls and prohibiting access to symptomatic workers are fundamental for preventing infections among farm animals.[8]

More research around this issue is needed, including larger-scale studies in different contexts, with animals from infected and noninfected homes and in areas with local transmission. To bridge this research gap, the OIE and the Food and Agriculture Organization (FAO) recommend public and animal health authorities undertake intersectoral risk analysis with a One Health approach. Meanwhile, international organizations suggest people testing positive for COVID-19 take precautions around animals, including isolating pets and other animals to avoid infection, while observing general hygiene measures with companion animals, in live animal markets and markets with animal products. $[1,2,8]$

In my opinion, the scientific community has sufficient evidence to support the hypothesis that animals are a potential link in the epidemiologic chain of human-human COVID-19 infection. Taking into account epidemiological antecedents and principles on the variability of disease processes in individuals and populations, depending on factors including the pathogens, hosts and environment, I consider the following steps necessary: 1) epidemiological studies of homes where COVID-19 is present should include variables related to pet and other animal ownership-number and type of pets/other animals in the home, their health status, human-animal relationship, where pets would go if the owner is hospitalized and 2) virology and/or serology tests for pets to detect SARS-CoV-2 infection. In resource-scarce settings, a case-con- 
trol study can be conducted, with protocols set by public health and veterinary services.

In Cuba, there are qualified veterinary medicine specialists to conduct this type of research; protocols to this effect proposed by the Animal Health Department, Ministry of Agriculture and the National Center for Agricultural Animal Health (CENSA) are currently being analyzed.
Many people maintain very close relationships with their pets, sometimes cohabitating with many animals (especially older people who live alone), while children enjoy playing with pets. Disseminating information about COVID-19 and animals will help promote recommended preventive measures. It's not about abandoning our pets, but rather taking all the necessary precautions to control the COVID-19 pandemic. $-1 /$ -

\section{REFERENCES}

1. OIE-World Organisation for Animal Health [Internet]. Paris: OIE-World Organisation for Animal Health; OIE 2020. Portal sobre COVID-19. Preguntas y respuestas sobre COVID-19; [updated 2020 Jun 9; cited 2020 Aug 20]. Available at: https://www.oie.int/es/nuestra-experiencia-cientifica/ informaciones específicas y recomendaciones/preguntas y respuestas del Covid-19/. Spanish.

2. Pan American Health Organization. Nota Informativa. Infecciones por SARSCoV-2 en animales [Internet]. Washington, D.C.: Pan American Health Organization; 2020 Jul 21 [cited 2020 Aug 20]. 5 p. Available at: http://docs.bvsalud .org/biblioref/2020/07/1103787/download.pdf. Spanish.

3. Hemida MG. Middle East Respiratory Syndrome Coronavirus and the One Health concept. PeerJ [Internet]. 2020 Aug 22 [cited 2020 Aug 24];7:e7556. Available at: http://doi.org/10.7717/peerj.7556

4. Shen M, Liu C, Xu R, Ruan Z, Zhao S, Zhang H, et al. SARS-CoV-2 infection of cats and dogs? Preprints [Internet]. Basel: MDPI; 2020 [cited 2020 Aug 20]. Available at: https://www.preprints.org/manuscript/202004.0116/v1

5. Xia X. Extreme genomic CpG deficiency in SARS-CoV-2 and evasion of host antiviral defense. Mol Biol Evol [Internet]. 2020 Apr 17 [cited 2020 Aug 20]. Epub ahead of print. Available at: https://academic.oup.com/mbe/article/ doi/10.1093/molbev/msaa094/58195
6. Zhang Q, Zhang H, Huang K, Yang Y, Hui X, Gao J, et al.. SARS-CoV-2 neutralizing serum antibodies in cats: a serological investigation. bioRxiv [Internet]. New York: Cold Spring Harbor Laboratory; 2020 Apr 3 [cited 2020 Apr 11]. Available at: https://doi.org/10.1101/2020.04.01.021196

7. Patterson EI, Elia G, Grassi A, Giordano A, Desario C, Medardo M, et al. Evidence of exposure to SARS-CoV-2 in cats and dogs from households in Italy. bioRxiv [Internet]. New York: Cold Spring Harbor Laboratory; 2020 Jul 23 [cited 2020 Aug 20]. Available at: https://www.biorxiv.org/content/10.1101/2020.07.2 1.214346v2.full.pdf

8. Food and Agriculture Organization of the United Nations (FAO). Guidelines to mitigate the impact of the COVID-19 pandemic on livestock production and animal health [Internet]. Rome: Food and Agriculture Organization of the United Nations (FAO); 2020 [cited 2020 Aug 30]. 19 p. Available at: http://www.fao .org/3/ca9177en/CA9177EN.pdf

Submitted: August 31, 2020

Approved for publication: September 11, 2020

Disclosures: None

Correspondence: percedo@censa.edu.cu, mipercedo@infomed.sld.cu 